\title{
Genotipos de rotavirus aislados de niños chilenos con gastroenteritis atendidos en dos hospitales públicos: variantes virales circulantes en un país con uso limitado de vacunas anti-rotavirus
}

\author{
Yalda Lucero, Nora Mamani, Héctor Cortés, Alfredo Peña, Rodrigo Vergara y Miguel O 'Ryan
}

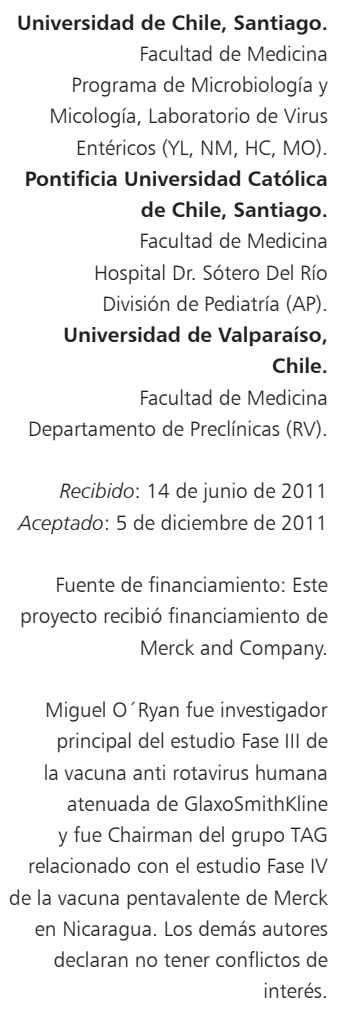

Recibido: 14 de junio de 2011 Aceptado: 5 de diciembre de 2011

Fuente de financiamiento: Este proyecto recibió financiamiento de Merck and Company.

Miguel O'Ryan fue investigador principal del estudio Fase III de

la vacuna anti rotavirus humana

atenuada de GlaxoSmithKline

y fue Chairman del grupo TAG

relacionado con el estudio Fase IV de la vacuna pentavalente de Merck

en Nicaragua. Los demás autores declaran no tener conflictos de interés.

Correspondencia a: Miguel O'Ryan Gallardo moryan@med.uchile.c

\section{Introducción}

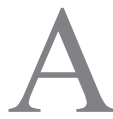
ctualmente rotavirus sigue siendo la principal causa de gastroenteritis (GE) grave en niños en todo el mundo, especialmente en países en vías de desarrollo ${ }^{1,2}$. Un estudio reciente realizado en nuestro país identificó a rotavirus como agente causal de $40 \%$ de las hospitalizaciones, $30 \%$ de las consultas a servicio de urgencia y $13 \%$ de las consultas en atención primaria por GE en niños bajo 5 años de edad ${ }^{3}$. Previamente se había estimado que cada año este agente causaría alrededor de 8.000 hospitalizaciones y 53.000 consultas a servicio de urgencia en niños bajo 3 años de edad ${ }^{4}$.

La respuesta inmune generada contra rotavirus luego de una infección natural o vacunación se ha correlacionado principalmente con la producción de anticuerpos neutralizantes contra las proteínas VP7 y VP4 que forman la cápside viral, si bien se ha propuesto un potencial rol antigénico de otras proteínas (VP6, NSP4)5. La proteína
VP7 es una glicoproteína para la cual se han descrito hasta ahora 23 tipos, denominados $\mathrm{G}$, de los cuales al menos 12 podrían causar enfermedad en humanos. VP4 por su parte, es una proteína sensible a proteasa, por lo que sus variantes se denominan $\mathrm{P}$ y hasta ahora se han descrito 32 tipos diferentes, de los cuales al menos 15 han sido encontrados en humanos ${ }^{5,6}$. La clasificación actual (genotipificación) de rotavirus se basa en el análisis de las secuencias de los genes que codifican para las proteínas VP7 (G) y VP4 (P), siendo las combinaciones genéticas predominantes en humanos en el mundo G1P[8], G2P[4], G3P[8], G4P[8] y G9P[8] $]^{6,7}$.

Recientemente se licenciaron en Chile dos vacunas anti-rotavirus que han demostrado una eficacia $>85 \%$ y una efectividad variable (45-80\%), siendo ésta mayor en países con mayor nivel de desarrollo ${ }^{6,8-10}$. Rotarix ${ }^{\circledR}$ (GlaxoSmithKline Biologicals, E.U.A.) es una vacuna compuesta por una cepa de rotavirus humano atenuado de genotipo G1P[8], mientras que Rota Teq ${ }^{\circledR}$ (Merck and Co., E.U.A.) es una vacuna compuesta por cinco cepas, 
reordenante bovino-humano, que contiene los genotipos humanos G1, G2, G3, G4 y P[8] ${ }^{6}$. Si bien la Organización Mundial de la Salud y otros organismos internacionales han recomendado priorizar la aplicación masiva de vacunas anti-rotavirus, especialmente en países en vías de desarrollo ${ }^{11}$ y estas vacunas han sido incorporadas a los programas nacionales de vacunación de, hasta ahora, 14 países de Latinoamérica ${ }^{6}$, nuestro país aún no ha adoptado esta política. En Chile, la vacunación contra rotavirus es de carácter voluntario a través del mercado privado, con una cobertura no determinada.

Ambas vacunas han demostrado proteger contra los genotipos humanos predominantes en estudios prelicenciamiento ${ }^{8,12,13}$; no obstante, no hay certeza absoluta para alguna de las dos vacunas de que esta protección sea equivalente para los diferentes genotipos, especialmente para genotipos que pudieran discrepar en uno o más antígenos de las cepas vacunales. Estudios de vigilancia de genotipos realizados luego de la incorporación de Rotarix ${ }^{\circledR}$ a los programas nacionales de inmunización de Brasil, Bélgica y Australia, han descrito un posible aumento en la proporción de casos causados por la variante G2P $[4]^{14-18}$, mientras que en Australia se ha informado de un posible aumento proporcional de G3P[8] luego de la incorporación de Rota Teq ${ }^{\circledR 18}$. No se ha dilucidado totalmente hasta ahora si estos cambios en el genotipo predominante se deben a una presión selectiva generada por estas vacunas o a fluctuaciones naturales, pues en períodos previos a que estuvieran disponibles estas vacunas y en países donde no han sido incorporadas de manera programática, también se habían descrito variaciones regionales y temporales del tipo viral más frecuente ${ }^{6,7}$. En nuestro país, en la década de los '90 se demostró un franco predominio de las variantes $\mathrm{G} 1$ en niños con $\mathrm{GE}^{19}$, mientras que estudios más recientes encontraron una mayor frecuencia, alrededor de $70 \%$, de las variantes $\mathrm{G} 4^{20,21}$.

Se deprende de lo antes mencionado la necesidad de realizar una vigilancia activa de los genotipos circulantes en una comunidad, antes, durante y después de la incorporación de vacunas anti-rotavirus a los programas nacionales de inmunización para evaluar la efectividad global y genotipo-específica de ellas así como para determinar las fluctuaciones de genotipos circulantes en ausencia o presencia del uso masivo de vacunas.

El objetivo de este trabajo fue proveer de información actualizada sobre los genotipos de rotavirus circulantes en Santiago y Valparaíso, representados por dos hospitales grandes de cada ciudad.

\section{Metodología}

\section{Pacientes}

Entre mayo 2009 y marzo 2010, se reclutaron niños bajo 5 años de edad, sin antecedente de vacunación anti- rotavirus, que consultaron por cuadro de GE en horario hábil al servicio de urgencia de los hospitales Dr. Sótero Del Río, de Santiago y Carlos van Buren, de Valparaíso. Para ello se contó con personal especialmente dedicado al estudio, encargado de identificar los niños con GE, solicitar consentimiento a los padres, y obtener una muestra de deposición. Se definió GE como $\geq 3$ deposiciones líquidas sin sangre $\mathrm{y} / \mathrm{o}<3$ deposiciones líquidas asociado $\mathrm{a} \geq 1$ vómito en un período de $24 \mathrm{hrs}$. Se registraron datos epidemiológicos y se tomó muestra de deposición recién emitida, de manera espontánea o luego de sondeo rectal, en frasco estéril. Las muestras fueron mantenidas a $-20^{\circ} \mathrm{C}$ hasta ser enviadas al Laboratorio de Virus Entéricos del Programa de Microbiología de la Facultad de Medicina, Universidad de Chile, para su posterior análisis.

Este protocolo fue aprobado por los comités de ética de investigación de los Servicios de Salud involucrados.

\section{Detección de rotavirus}

Todas las muestras de deposición obtenidas fueron evaluadas mediante kit de ELISA comercial de acuerdo a las instrucciones de los proveedores (IDEIA-Rotavirus ${ }^{\circledR}$, OXOID, Cambridge, R. Unido).

\section{Genotipificación de rotavirus}

Se extrajo ARN de las muestras positivas para rotavirus mediante método Trizol LS ${ }^{\circledR}$ (Invitrogen, E.U.A.) de acuerdo a las instrucciones del proveedor. Se amplificaron segmentos de los genes VP7 y VP4 mediante partidores específicos utilizando una reacción de polimerasa en cadena asociada a transcripción reversa (RPC-TR) de tipo semi-anidada, como se ha descrito previamente ${ }^{22}$. En breve, se realizó un primer ciclo de RPC utilizando los partidores VP7-F y VP7-R para el gen VP7, y Con-3 y Con-4 para el gen VP4. Luego se hizo un segundo ciclo de RPC utilizando partidores genotipo-específicos en dirección río arriba y VP7-R y Con-4 como partidores reversos para los genes VP7 y VP4, respectivamente. Este método permite identificar los genotipos G1, G2, G3, G4, G8, G9 y G10 de VP7, además de P[4], P[6], P[8], P[9], $\mathrm{P}[10]$ y $\mathrm{P}[11]$ de VP4.

\section{Análisis de datos}

Se registró en formulario ad-hoc la identificación y edad de cada paciente, además de la fecha de cada episodio. Estos datos, junto con el número de muestras positivas para rotavirus y los genotipos virales fueron ingresados a base de datos generada en programa Excel ${ }^{\circledR}$. Los datos fueron analizados mediante programa estadístico EpiInfo 3.5.3 ${ }^{\circledR}$ (CDC, Atlanta, E.U.A.), utilizando para las variable continuas el test ANOVA y para las variables discretas los test de $\chi^{2}$ o test exacto de Fisher según correspondiera. 


\section{Resultados}

\section{Detección de rotavirus}

Durante el período de 11 meses de este estudio se analizó un total de 967 muestras, de las cuales 296 (31\%) fueron positivas para rotavirus por ELISA. La proporción de casos positivos fue mayor en el Hospital Sótero Del Río, siendo en este centro de 33\% (213/643) mientras que en el Hospital Carlos van Buren fue de 26\% (83/324) $(p=0,016)$. La mediana de edad de los casos evaluados en el Hospital Sótero Del Río fue menor que la de los niños atendidos en el Hospital Carlos van Buren (mediana 15 y 17 meses, respectivamente, $p=0,007)$. No se encontraron diferencias en la proporción de pacientes hospitalizados entre los dos centros (12 y 16\%, respectivamente, $p>0,05$ ).

En la Tabla 1 se muestran las características epidemiológicas de los pacientes con y sin infección por rotavirus. La mediana de edad de los pacientes en los que se detectó rotavirus fue significativamente menor que la de aquellos en quienes no se identificó este agente ( 13 y 16 meses, respectivamente, $p<0,01)$. Hubo una mayor proporción de pacientes con infección por rotavirus en el rango 7-24 meses que en los otros grupos de edad (31\%, 34\% y $21 \%$ para los grupos 0-6 meses, 7-24 meses y 25-60 meses, respectivamente), lo que resultó ser estadísticamente significativo (OR 1,8 al comparar infección por rotavirus en el grupo 7-24 meses con los otros grupo de edad; IC 95\%: 1,3-2,4; $p<0,001)$. No se encontraron diferencias en cuanto a la distribución de género $(\mathrm{p}=0,87)$.

Se identificaron casos de infección por rotavirus du- rante todo el período de estudio, con una mediana global de 22 casos por mes. Se evidenció un pico de frecuencia de GE por esta etiología en los meses de noviembre y diciembre, en los que prácticamente se triplicó el número de casos por mes (60 y 58 casos en noviembre y diciembre, respectivamente, $p<0,001$ al comparar con el resto del período) (Figura 1A). Al analizar los centros por separado, este marcado aumento de casos en noviembre/ diciembre se evidenció con claridad en el Hospital Sótero Del Río; no así en el Hospital Carlos van Buren, donde la distribución de casos fue más homogénea $(\mathrm{p}<0,001$ para la comparación de distribución de casos de ambos hospitales) (Figura 1B y 1C).

\section{Genotipos de rotavirus encontrados}

Se logró realizar con éxito genotipificación para los genes VP7 y VP4 en 206/296 muestras. En los casos restantes no se contó con suficiente muestra de deposición o no se logró la amplificación de los genes mencionados. Las combinaciones de genotipos encontrados para VP7 (G) y VP4 (P) se muestran en la Tabla 2.

El genotipo G9P [8] predominó ampliamente durante todo el período de estudio, tanto en Valparaíso como en Santiago, seguido de G1P[8] y G2P[4] (Figura 1, Tabla 2).

La distribución temporal de casos causados por rotavirus G9P [8] tuvo una curva similar, aunque de menor magnitud, a la de casos totales de GE por rotavirus. $\mathrm{Si}$ bien se encontró una mayor frecuencia de G9P[8] en los meses noviembre y diciembre, este aumento de casos no resultó significativo.

Tabla 1. Características epidemiológicas de 967 niños bajo 5 años de edad con gastroenteritis (con y sin infección por rotavirus) atendidos en dos hospitales pediátricos chilenos

\begin{tabular}{|c|c|c|c|c|}
\hline & Total & Con infección por rotavirus & Sin infección por rotavirus & $\mathbf{p}$ \\
\hline $\mathrm{N}^{\circ}$ de pacientes & 967 & 296 & 671 & \\
\hline Mediana de edad (rango) & 15 meses (0-59 meses) & 13 meses ( $1-58$ meses) & 16 meses (0-60 meses) & $<0,01$ \\
\hline \multicolumn{5}{|c|}{$\mathrm{N}^{\circ}$ pacientes según grupo de edad (\%) } \\
\hline $0-6$ meses & 158 & $50(31 \%)$ & $108(69 \%)$ & $<0,01^{\dagger}$ \\
\hline 7-24 meses & 582 & $198(34 \%)$ & $384(66 \%)$ & $<0,01^{\ddagger}$ \\
\hline $25-60$ meses & 227 & $48(21 \%)$ & $179(79 \%)$ & \\
\hline $\mathrm{N}^{\circ}$ varones (\% respecto al total) & $559(58 \%)$ & $170(58 \%)$ & $389(58 \%)$ & $>0,05$ \\
\hline \multicolumn{5}{|l|}{ Proporción de casos por trimestre } \\
\hline Mayo-julio & 175 & $56(32 \%)$ & $119(68 \%)$ & $<0,001^{*}$ \\
\hline Agosto-octubre & 303 & $80(26 \%)$ & $223(74 \%)$ & \multirow[t]{3}{*}{$<0,001^{* *}$} \\
\hline Noviembre-enero & 340 & $138(45 \%)$ & $202(55 \%)$ & \\
\hline Febrero-marzo & 149 & $22(15 \%)$ & $127(85 \%)$ & \\
\hline
\end{tabular}




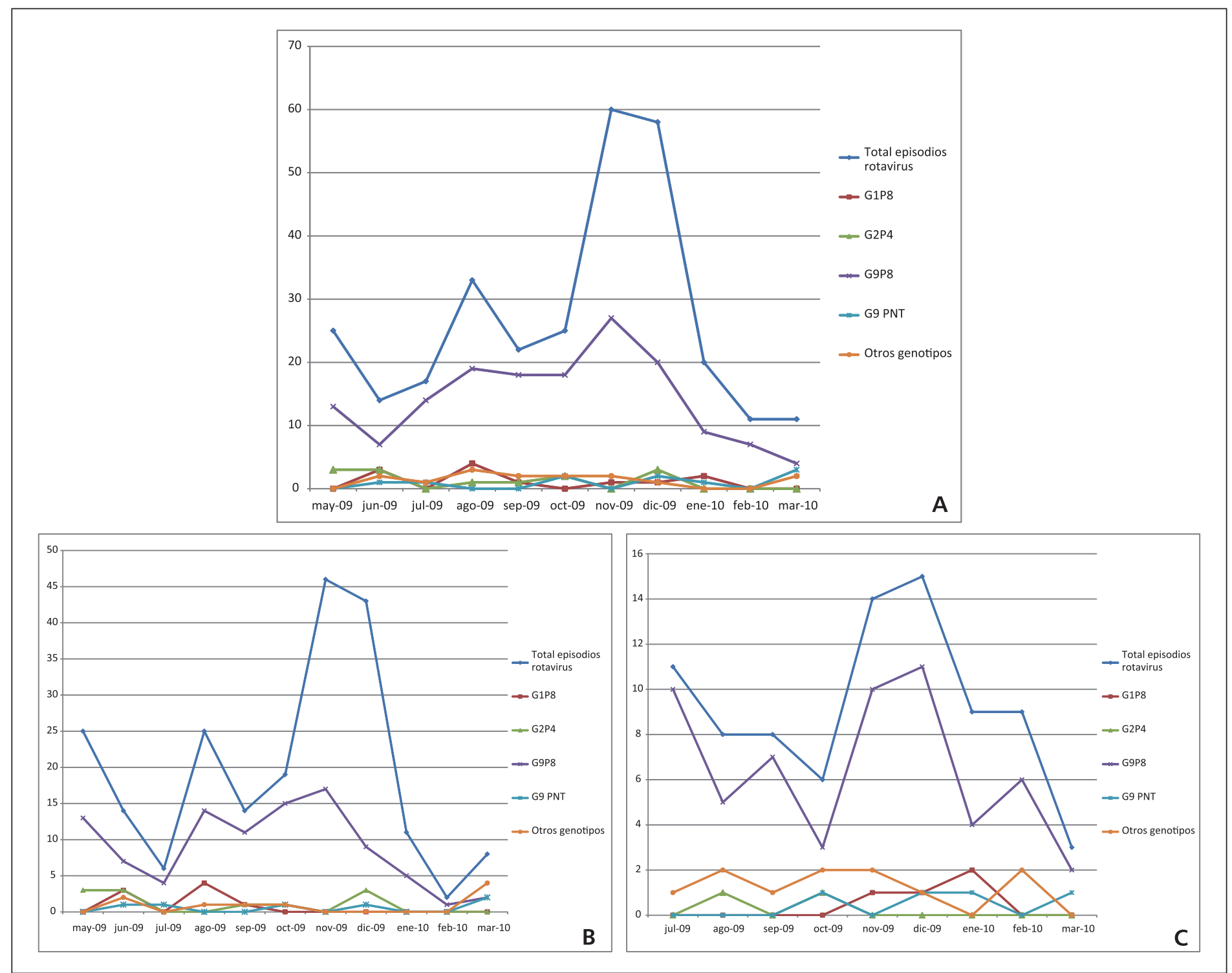

Figura 1. Distribución mensual de episodios de gastroenteritis por rotavirus. A. Total de casos de rotavirus y número de casos según genotipo viral. B. Distribución de casos totales y según genotipo viral en Hospital Sótero del Río. C. Distribución de casos totales y según genotipo viral en Hospital Carlos van Buren.

El predominio de G9P[8] respecto de las otras variantes de rotavirus fue transversal a todos los grupos de edad evaluados. Sin embargo, los lactantes de 0-6 meses presentaron una tendencia a una mayor diversidad de genotipos (Tabla 2) ( $\mathrm{p}=0,18$ y 0,41 al comparar la frecuencia de G9P [8] en el grupo 0-6 meses con los grupos 7-24 y 25-60 meses, respectivamente).

\section{Discusión}

Rotavirus fue identificado en aproximadamente $1 / 3$ de los casos de GE estudiados, lo que concuerda con
Tabla 2. Genotipos virales G (VP7) y P (VP4) de rotavirus encontrados en pacientes bajo 5 años de edad, con gastroenteritis atendidos en dos hospitales chilenos

\begin{tabular}{|lrrrrrr} 
& Total & G1P[8] & G2P[4] & G9P[8] & G9P[NT $]^{*}$ & \multicolumn{1}{c}{$\begin{array}{c}\text { Otros } \\
\text { genotipos }^{* *}\end{array}$} \\
\hline$N^{\circ}$ total de pacientes (\%) & 206 & $12(6 \%)$ & $13(6 \%)$ & $156(76 \%)$ & $10(5 \%)$ & $15(7 \%)$ \\
Según rango de edad & & & & & & \\
$\quad$ 0-6 meses (\%) & 44 & $3(7 \%)$ & $1(2 \%)$ & $29(66 \%)$ & $1(2 \%)$ & $10(23 \%)$ \\
$7-24$ meses (\%) & 131 & $6(4 \%)$ & $9(7 \%)$ & $103(79 \%)$ & $9(7 \%)$ & $4(3 \%)$ \\
$25-60$ meses (\%) & 31 & $3(10 \%)$ & $3(10 \%)$ & $24(77 \%)$ & 0 & $1(3 \%)$ \\
\hline
\end{tabular}

${ }^{*} \mathrm{NT}=$ no tipificable. ${ }^{* *}$ Incluye G4P[8], G2P[NT], G1P[6], G1P[NT], G3P[8], G3P[NT]. ${ }^{* * *} p=0,04$ para comparación frecuencia G9P[8] en grupo 0-6 meses vs 7-24 meses. 
lo descrito previamente en la literatura científica ${ }^{1,4,6}$. En un meta-análisis reciente de Linhares y cols., sobre la epidemiología de rotavirus en Latinoamérica y el Caribe, se describió un promedio de $25,1 \%$ para rotavirus como causa de GE en niños bajo 5 años de edad, levemente inferior a la reportada en nuestra serie ${ }^{6}$. Esta mayor proporción encontrada por nuestro grupo podría deberse a las mejores condiciones sanitarias de Chile comparado con otros países de la región, lo que condicionaría una disminución de los casos de GE bacteriana y parasitaria y un aumento proporcional de las etiologías virales, o a que el presente estudio sólo incluyó pacientes atendidos en hospitales, lo que seleccionaría casos de mayor gravedad, habitualmente relacionados con rotavirus ${ }^{3}$. Sin embargo, en el diseño de nuestro estudio no se incluyó la detección de otros agentes etiológicos por lo que estas hipótesis son meramente especulativas.

Llamó la atención que la proporción de muestras en las que se identificó rotavirus fue significativamente mayor en los pacientes atendidos en el Hospital Sótero Del Río, de Santiago, que en aquellos evaluados en el Hospital Carlos van Buren, de Valparaíso. Esto podría explicarse, al menos en parte, por las diferencias encontradas en la distribución etárea, o al posible efecto de condiciones sanitarias y/o climáticas diferentes y/o, menos probablemente, a una disminución de la detección de rotavirus en las muestras procedentes del hospital más lejano debido a degradación de las partículas virales durante su transporte.

Se encontró una proporción significativamente mayor de casos de rotavirus en noviembre-diciembre, fenómeno que sólo se evidenció en el hospital ubicado en Santiago. Esto contrasta con la mayor incidencia invernal descrita clásicamente en países con clima templado, como el nuestro ${ }^{23,24}$. Se desconoce el motivo de este comportamiento epidemiológico diferente tanto en relación a los estudios previos como entre las dos zonas estudiadas. Se requeriría un período de vigilancia mayor para definir con mayor claridad si esta variación en la estacionalidad de rotavirus es real o un artefacto de muestreo.

La mayor parte de los casos se presentó en lactantes de 7 a 24 meses de edad. Esto concuerda con lo descrito por Linhares y cols., en niños latinoamericanos ${ }^{6}$. Este sería el grupo más susceptible y el que se beneficiaría más con el eventual uso de una vacuna anti-rotavirus administrada durante los primeros 6 meses de vida.

Es destacable el claro predominio evidenciado para el genotipo G9P[8], que se identificó aproximadamente en 2 de cada 3 casos en lactantes con infección rotaviral de 0-6 meses y 3 de cada 4 niños de 7-60 meses. Estudios realizados en nuestro país a mediados de la década de los ' 90 describieron un predominio de las variantes G1, especialmente $\mathrm{G} 1 \mathrm{P}[8]^{19}$, mientras que reportes posteriores, de mediados de la década pasada, evidenciaron una mayor frecuencia de las variantes G4 ${ }^{20,21}$. Estas variaciones en el genotipo predominante serían producto de fluctuaciones naturales e independientes del potencial efecto selectivo de las vacunas anti-rotavirus.

El genotipo G9P[8] se describió por primera vez en 1983 en Pensilvania, E.U.A. y mantuvo una representación baja como causa de GE hasta hace aproximadamente 10 años, en que aumentó su frecuencia en el mundo, considerándose actualmente como uno de los genotipos de circulación más frecuente luego de G1[P8], G2[P4], G3[P8], G4[P8] ${ }^{25}$. Linhares y cols., informaron en el metaanálisis descrito más arriba, un promedio de $8,8 \%$ de GE en niños bajo 5 años de edad causadas por el genotipo G9P[8], considerando en conjunto los datos de 43 estudios realizados en países de Latinoamérica y el Caribe ${ }^{6}$. En ese estudio, G9P[8] resultó ser el tercer genotipo más frecuente, luego de G1P[8] y G2P[4].

Si bien, hasta ahora han sido muy escasos los reportes en que G9P [8] ha sido la variante de rotavirus predominante, los datos sugieren que su frecuencia podría ir en aumento ${ }^{25-28}$. Abdel-Haq y cols., describieron recientemente en niños norteamericanos con GE un 20\% de G9P[8], siguiendo muy de cerca, como segundo genotipo en frecuencia, a G1P[8] $(21 \%)^{26}$. Por su parte Esteban y cols., en Argentina, y Ribas y cols., en Cuba, reportaron en niños con GE 30\% y hasta $78 \%$ de rotavirus G9P[8], respetivamente, siendo en ambos casos el genotipo más frecuente ${ }^{27,28}$.

El genotipo G9 no está incluido en las dos vacunas actualmente disponibles. Sin embargo los estudios prelicencia de dichas vacunas demostraron una disminución de los casos de GE por variantes G9P [8], con una eficacia de $65-85 \%{ }^{12,13}$. Esto podría ser explicado, al menos en parte, por la presencia de anticuerpos neutralizantes contra el antígeno $\mathrm{P}[8] \mathrm{y} / \mathrm{o}$ por reacción cruzada frente a antígenos $\mathrm{G}$ u otros antígenos de la cápside interna o de proteínas no estructurales. Esto sugiere que, de acuerdo a la distribución de genotipos encontrada en este estudio, la incorporación programática de alguna de las vacunas anti-rotavirus disponibles actualmente, podría tener un impacto significativo en la disminución de casos de GE.

Recientemente se ha descrito un aumento de la circulación de la variante G12, no incluida en el protocolo de genotipificación utilizado en este estudio ${ }^{6,29,30}$. De acuerdo al meta-análisis realizado por Linhares y cols., hasta 5\% de las GE por rotavirus en niños latinoamericanos estaría siendo causado por variantes con VP7 tipo G12 ${ }^{6}$. Sin embargo, algunos autores han descrito una proporción bastante mayor de casos por esta variante; Sherchand y cols., describieron una proporción de hasta $50 \%$ de los casos de GE causadas por esta variante en niños de $\mathrm{Nepal}^{29}$. En nuestro estudio no se logró genotipificar un 30\% de las muestras mediante los partidores utilizados. Al menos una parte de éstas podrían corresponder al genotipo G12, por lo que sería deseable evaluar en este grupo de muestras no tipificables la presencia de este genotipo emergente. 
Esto es particularmente relevante, considerando que hasta ahora no se ha determinado la eficacia de las vacunas anti-rotavirus contra esta variante viral.

En conjunto, los resultados de este estudio y reportes de vigilancias previas, ponen en evidencia la evolución dinámica de la epidemiología molecular de rotavirus y resaltan la necesidad de mantener redes de vigilancia de los genotipos circulantes para determinar la variación natural de estos, la posible influencia en esta variación del uso masivo de vacunas, y la hipotética posibilidad del surgimiento puntual de genotipos no cubiertos por una u otra vacuna. De acuerdo a nuestros resultados, la mayor incidencia de GE por rotavirus estaría justamente en el rango de edad susceptible de ser protegido por vacunación (7-24 meses) y de acuerdo a los genotipos circulantes, las vacunas actualmente disponibles podrían tener un impacto positivo en disminuir los casos graves de GE en este grupo.

Agradecimientos: Agradecemos el riguroso trabajo de las Sras. Laura Muñoz y Erika Farías, que realizaron el reclutamiento de pacientes y recolección de muestras clínicas en los hospitales Carlos van Buren y Sótero del Río, respectivamente.

\section{Resumen}

Antecedentes: Rotavirus es la principal causa de gastroenteritis (GE) grave en niños. Actualmente se dispone de dos vacunas con eficacia demostrada contra los genotipos predominantes en el mundo. Los genotipos de rotavirus varían en el tiempo y de una región a otra. Es importante mantener la vigilancia de los genotipos circulantes para monitorizar las tendencias asociadas o no al uso de vacunas. Objetivo: Actualizar la información sobre genotipos de rotavirus circulantes en dos ciudades importantes de Chile (Santiago y Valparaíso). Metodología: Entre mayo 2009 y marzo 2010 se reclutaron niños bajo 5 años de edad con GE atendidos en dos hospitales; ninguno de ellos con historia previa de vacunación antirotavirus. Se registró información epidemiológica y se tomó muestra de deposición para detección de rotavirus mediante ELISA comercial. Se realizó genotipificación mediante RPC-TR semi-anidada. Resultados: Se detectó rotavirus en 296/967 muestras analizadas (31\%), con un pico de frecuencia en noviembre/diciembre y afectando predominantemente al grupo de 7-24 meses de edad (67\%). G9P[8] fue el genotipo predominante (76\%), seguido por G1P[8] (6\%) y G2P[4] (6\%) en ambas ciudades. Conclusiones: Rotavirus causó un tercio de las GE en este grupo, afectando especialmente al grupo de edad que podría beneficiarse con la vacunación antirotavirus. G9P [8], una de las variantes contra las cuales las vacunas antirotavirus han demostrado alta eficacia, fue por lejos el genotipo más frecuente. Es necesario continuar la vigilancia en Chile de modo de conocer el impacto de la enfermedad y diversidad de variantes antes de la incorporación de una vacuna anti-rotavirus.

\section{Referencias}

1.- Parashar U, Gibson C, Bresee J, Glass R. Rotavirus and severe childhood diarrhea. Emerg Infect Dis 2006; 12: 304-6.

2.- Parashar U, Burton A, Lanata C, Boschi-Pinto C, Shibuya K, Steele D, et al. Global mortality associated with rotavirus disease among children in 2004. J Infect Dis 2009; 200: S9-15.

3.- O’Ryan M, Peña A, Vergara R, Díaz J, Mamani N, Cortés H, et al. Prospective characterization of norovirus compared with rotavirus acute diarrhea episodes in Chilean children. Pediatr Infect Dis J 2010; 29: 855-9.

4.- O'Ryan M, Pérez-Schael I, Mamani N, Peña A, Salinas B, González G, et al. Rotavirusassociated medical visits and hospitalizations in South America: a prospective study at three large sentinel hospitals. Pediatr Infect Dis J 2001; 20: 685-93.

5.- Kirkwood C. Genetic and antigenic diversity of human rotaviruses: potential impact on vaccination programs. J Infect Dis 2010; 202: S43-48.

6.- Linhares A, Stupka J, Ciapponi A, Bardach A, Glujovsky D, Aruj P, et al. Burden and typing of rotavirus group A in Latin America and the Caribbean: systematic review and meta-analysis. Rev Med Virol 2011; doi: 10.1002/rmv.682.

7.- Santos N, Hoshino Y. Global distribution of rotavirus serotypes/genotypes and its implication for the development and implementation of an effective rotavirus vaccine. Rev Med Virol 2005; 15: 29-56.

8.- O'Ryan M, Hermosilla G, Osorio G. Rotavirus vaccines for the developing world. Curr Opin Infect Dis 2009; 22: 483-9.

9.- Hull J, Tell E, Kerin T, Freeman M, Esona M, Gentsch J, et al. United States rotavirus strain surveillance from 2005 to 2008 : genotype prevalence before and after vaccine introduction. Pediatr Infect Dis J 2011; 30: S42-S47.

10.- Madhi S, Cunliffe N, Stelle D, Witte D, Kirsten M, Louw C, et al. Effect of human rotavirus vaccine on severe diarrhea in African infants. N Engl J Med 2010; 362: 289-98.

11.- Glass R, Parashar U, Bresee J, Turcios R, Fischer T, Widdowson M, et al. Rotavirus vaccines: current prospects and future challenges. Lancet 2006;368: 323-32.

12.- Ruiz-Palacios G, Pérez-Schael I, Velásquez R,
Abate H, Breuer T, Costa S, et al. Safety and efficacy of an attenuated vaccine against rotavirus severe gastroenteritis. N Engl J Med 2006;354: 11-22.

13.- Vesikari T, Matson D, Denehi P, Van Damme P, Santosham M, Rodríguez Z. Safety and efficacy of a pentavalent human-bovine (WC3) reassortant rotavirus vaccine. N Engl J Med 2006; 354: 23-33.

14.- Gurgel R Q, Correia J B, Cuevas L E. Effect of rotavirus vaccination on circulating virus strains. Lancet 2008; 371: 301-2

15.- Nakagomi T, Cuevas L E, Gurgel R, Elrokhi S H, Belkhiv Y A, Abugalia M, et al. Apparent extinction of non-G2 rotavirus strains from circulation in Recife, Brazil, after the introduction of rotavirus vaccine. Arch Virol 2008; 153: 591-3

16.- Sáfadi M A, Berezin E N, Munford V, Almeida F J, de Moraes J C, Pinheiro C F, et al. Hospital-based surveillance to evaluate the impact of rotavirus vaccination in São Paulo, Brazil. Pediatr Infect Dis J 2010; 29, 1019-22.

17.- Zeller M, Rahman M, Heylen E, De Coster S, De Vos S, Arijs I, et al. Rotavirus incidence and 
genotype distribution before and after national rotavirus vaccine introduction in Belgium.

Vaccine 2010; 28: 7507-13.

18.- Kirkwood C D, Boniface K, Barnes G L, Bishop R F. Distribution of rotavirus genotypes after introduction of rotavirus vaccines, Rotarix and RotaTeq, into the national immunization program of Australia. Pediatr Infect Dis J 2011; 30: S48-S53.

19. O'Ryan M, Mamani N, Avendaño L, Cohen J, Peña A, Villarroel J, et al. Molecular epidemiology of human rotaviruses in Santiago, Chile. Pediatr Infect Dis J 1997; 16: 305-11.

20.- Vergara R, Navarrete M, Núñez E, Escobar L, Navarro S, Venegas G, et al. Impacto de gastroenteritis severa por rotavirus en niños chilenos menores de 3 años de edad. Rev Med Chile 2007; 135: 975-81.

21.- O’Ryan M, Díaz J, Mamani N, Navarrete M, Vallebuono C. Impact of rotavirus infections on outpatient clinic visits in Chile. Pediatr Infect Dis J 2007; 26: 41-5.
22.- Iturriza-Gomara M, Kang G, Gray J. Rotavirus genotyping: keeping up with an evolving population of human rotaviruses. J Clin Virol 2004; 31: 259-65.

23.- Cook S, Glass R, LeBaron C, Ho M. Global seasonality of rotavirus infections. Bull World Health Organ 1990; 68 (2): 171-7.

24.- Kane E, Turcios R, Arvay M, García S, Bresee J, Glass R. The epidemiology of rotavirus diarrhea in Latin America. Anticipating rotavirus vaccines. Rev Panam Salud Pública 2004; 16: 371-7.

25.- O'Ryan M. The ever changing landscape of rotavirus serotypes. Pediatr Infect Dis J 2009; 28: S60-S62.

26.- Abdel-Haq N, Amjad M, McGrath E, Chearskul P, Amer A, Salimnia H, et al. Emergence of human rotavirus genotype G9 in Metropolitan Detroit between 2007 and 2009. J Med Microbiol 2011; 60: 761-7.

27.- Esteban L, Rota R, Gentsch J, Jiang B, Esona M, Glass R, et al. Molecular epidemiology of group A rotavirus in Buenos Aires, Argentina 2004-2007: Reemergence of G2P [4] and emergence of G9P [8] strains.

J Med Virol 2010; 82: 1083-93.

28.- Ribas M, Nagashima S, Calzado A, Acosta G, Tejero Y, Cordero Y, et al. Emergence of G9 as a predominant genotype of human rotaviruses in Cuba. J Med Virol 2011; 83: 738-44.

29.- Sherchand J, Nakagomi O, Dove W, Nakagomi T, Yokoo M, Pandey B, et al. Molecular epidemiology of rotavirus diarrhea among children aged $<5$ years in Nepal: Predominance of emergent G12 strains during 2 years. J Infect Dis 2009; 200: S182-7.

30.- Iturriza-Gómara M, Dallman T, Bányai K, Böttiger B, Buesa J, Diedrich S, et al. Rotavirus genotypes co-circulating in Europe between 2006 and 2009 as determined by EuroRotaNet, a pan-European collaborative strain surveillance network. Epidemiol Infect 2011; 139: 895-909. 